\title{
Metz
}

Parc urbain de la Seille

\section{Emmanuelle Miejac}

\section{(2) OpenEdition}

12 Journals

Édition électronique

URL : http://journals.openedition.org/adlfi/8726

ISSN : 2114-0502

Éditeur

Ministère de la culture

Référence électronique

Emmanuelle Miejac, « Metz », ADLFI. Archéologie de la France - Informations [En ligne], Lorraine, mis en ligne le 01 mars 2001, consulté le 20 avril 2019. URL : http://journals.openedition.org/adlfi/8726

Ce document a été généré automatiquement le 20 avril 2019.

(c) Ministère de la Culture et de la Communication, CNRS 


\section{Metz}

Parc urbain de la Seille

\section{Emmanuelle Miejac}

Identifiant de l'opération archéologique : F1357200100086

Date de l'opération : 2001 (SU)

Succédant à un campagne de sondages de diagnostic (voir Bilan scientifique régional- 2000, p. 87-88), les fouilles préventives menées dans le cadre de l'aménagement du parc urbain de la Seille ont permis d'étudier des aménagements de berge de la Seille. Entre 935-952 un renfort de berge est installé sur les bords de la rivière hors des murs de la ville de Metz; une zone particulière était probablement dédiée à l'accostage des bateaux (ensemble poutre-pieux au sud de la zone 2). Des destructions puis réfections se succèdent rapidement jusqu'en 985. Les principes de construction de ce premier ensemble sont simples : rangées de gros pieux de chêne assez éloignés associés à une file de petits pieux de hêtre soutenant un clayonnage. Les réparations ont été réalisées après l'affaissement de la structure par un empierrement et de nouvelles files de pieux. Le clayonnage, composé de piquets de chêne verticaux et de branchages de noisetier horizontaux, renforce de nouveau la structure.

La construction semble s'être développée depuis le nord jusqu'au sud de la zone de fouille. Alors qu'au sud, elle s'installait dans le lit vif, au nord, elle prenait appui sur la berge est. La partie sud du site est installée en un lieu ou le courant était moins fort, à l'envasement plus important. La Seille, par sa faible pente, semble avoir toujours connu un envasement récurrent - apparition d'atterrissements, divagation de son cours - qui ne favorisa ni la navigation, ni l'exploitation de ces berges. Ce renfort servait également à maintenir le cours d'eau vers le coteau est. Les raisons de l'implantation de cette structure semblent être à la fois environnementales et économiques : création dès le $\mathrm{X}^{\mathrm{e}} \mathrm{s}$. d'un chenal fixe, permettant une petite navigation locale (exploitation des gisements de sel), un début de contrôle des divagations de la rivière et de ses crues. Cela favorisa le dégagement de nouveaux terrains à exploiter. 
Vers 984-985, une construction en aval de ce premier ensemble est installée. Il s'agit d'un barrage oblique utilisant uniquement des pieux de chêne. Des réfections furent également faites l'année suivant sa première implantation. Cet ensemble était utilisé pour conduire le courant vers la berge ouest. Entre cette construction et la berge, un espace devait être laissé libre, soit pour l'installation d'un moulin soit pour celle de nasses et/ou filets. La preuve de cette fonction n'a pu être apportée puisque la liaison entre la berge et cet élément n'a pas été retrouvée, la limite de fouille ne le permettant pas. En aval de cette construction, un appontement en bois devait être construit à l'aide de pieux de forts diamètres. L'accostage des bateaux pourrait être mis en relation avec l'élément installé entre la berge et le barrage, moulin, pêcherie?

$\mathrm{Au} \mathrm{X}^{\mathrm{e}} \mathrm{s}$., les ecclésiastiques s'intéressaient particulièrement à l'aménagement des domaines humides. L'une des abbayes du Sablon pourrait être à l'origine de l'installation des différentes constructions. Le renfort de berge, permettant de rendre salubre et exploitable une zone plus ou moins marécageuse, souvent recouverte par les inondations, matérialisait dans le même temps une zone d'accostage pour les échanges avec les terres de l'amont, l'exploitation des salines et des terres agricoles. Cela permettait donc d'assurer l'approvisionnement de l'abbaye et de ce secteur du Sablon occupé de façon importante au $\mathrm{X}^{\mathrm{e}} \mathrm{s}$., mais aussi peut-être de la cité.

L'abandon total après 985 de ces aménagements marque un événement d'importance dans ce secteur, recul de pouvoir de l'abbaye sur son environnement, recul des échanges? Cet abandon pose également le problème du déplacement de lieux de chargement et déchargement vers la ville, de l'équilibre des pouvoirs, du développement économique de la région, de la formation du tissu urbain. Un port a existé et a perduré au niveau du boulevard Paixhans; celui-ci aurait-il pris l'avantage dans le commerce sur la Seille, associé à celui localisé au niveau de la place de la Comédie sur la Moselle? Ces questions ne pourront être résolues que par des données recueillies sur de nouvelles fouilles, dans la ville de Metz mais également sur tout le cours de la Seille afin d'évaluer l'exploitation et son évolution.

(Fig. $n^{\circ} 1$ : Plan général des aménagements du $X^{\mathrm{e}}$ s.), (Fig. $\mathbf{n}^{\circ} \mathbf{2}$ : Vue d'ensemble du renfort de berge) et (Fig. $n^{\circ} 3$ : Détail du renfort de berge)

\section{ANNEXES}


Fig. $n^{\circ} 1$ : Plan général des aménagements du $X^{e} s$.

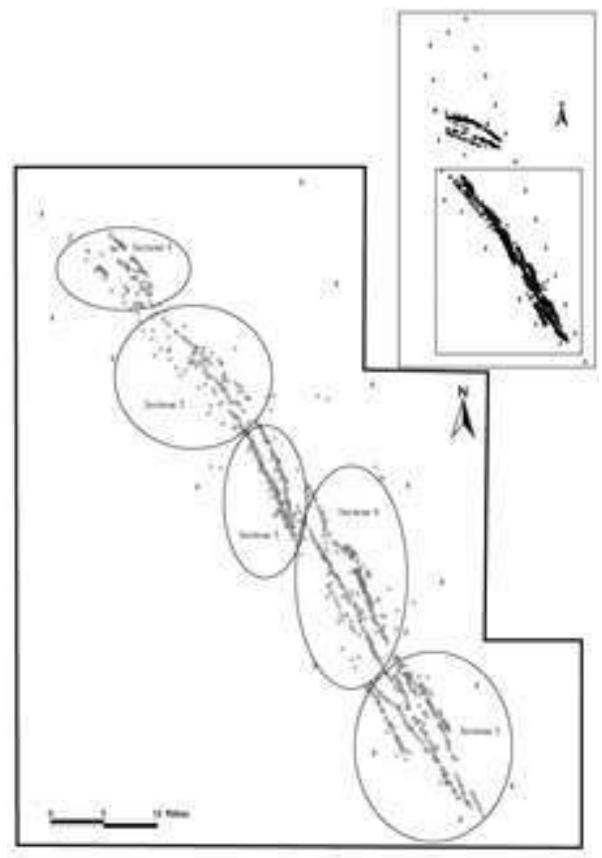

INRAP (2001)

Fig. $n^{\circ} 2$ : Vue d'ensemble du renfort de berge

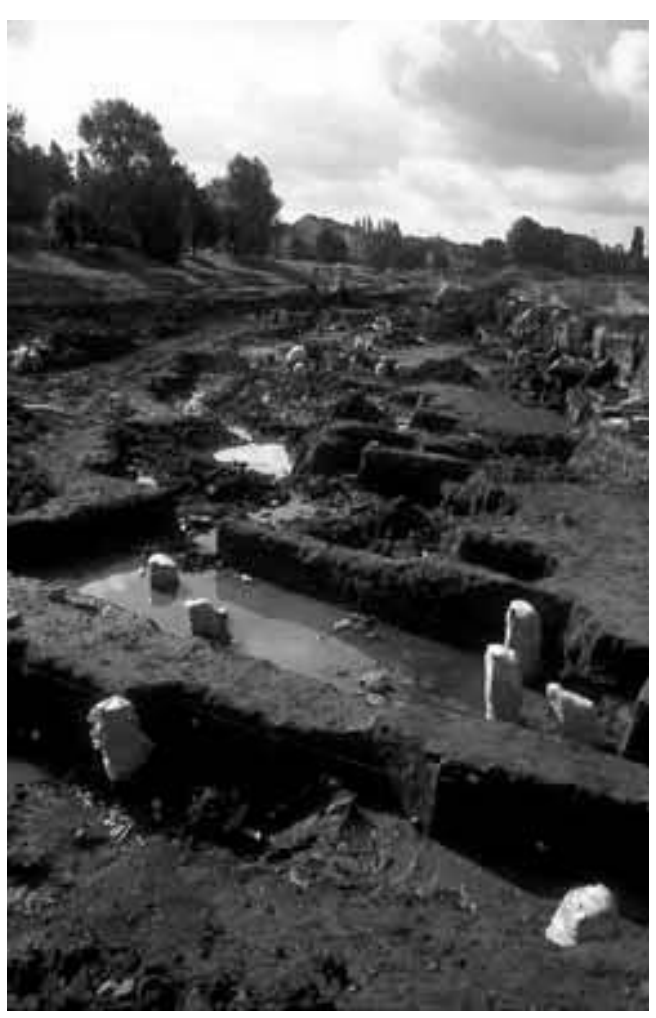

INRAP (2001) 
Fig. $n^{\circ} 3$ : Détail du renfort de berge

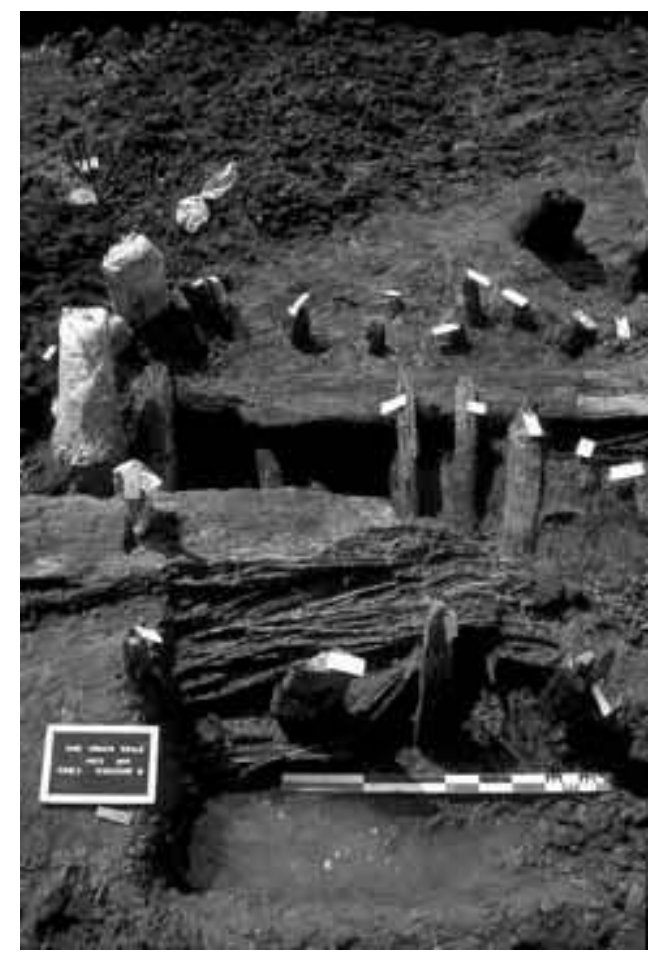

$\operatorname{INRAP}(2001)$

\section{INDEX}

Index géographique : Lorraine, Moselle (57), Metz operation Sauvetage urgent (SU)

Index chronologique : Moyen Âge*, Moyen Âge

Thèmes : abbaye, aménagement de berge, appontement, barrage, chenal, développement économique, filet, moine, moulin, nasse, pêche, pêcheur, pieu, port

\section{AUTEURS}

\section{EMMANUELLE MIEJAC}

AFAN 